Ecole d’Eté Systèmes Optiques

\title{
Les imageurs spectraux
}

\author{
R. Damaschini
}

Laboratoire Aimé Cotton, CNRS II, Bat. 505, Campus d'Orsay, F-91405 Orsay cedex, France

Résumé : Un imageur spectral est un instrument qui fournit le spectre de chaque pixel. Il est utilisé pour l'observation de la terre depuis l'espace (spectro-télescope) et pour l'analyse des surfaces (spectro-microscope). Trois types d'imageur spectral utilisant la spectroscopie dispersive et la réception sur matrice de récepteurs sont décrits et comparés du point de vue du rapport signal sur bruit.

Abstract : A spectral imaging device is a device which gives the spectral content for each pixel. It is used to terrestrial environment study and to surface analysis. Three types of spectral imaging device based on dispersive spectroscopy and reception on photodiode array are described and compared one another with regard to signal-to-noise ratio. 


\section{INTRODUCTION}

La notion d'image nous est familière. L'oeil en est le récepteur privilégié et l'imagerie traditionnelle en tient le plus grand compte.

Mais dans certains domaines le chercheur ou l'ingénieur a besoin d'une information plus précise. Le spectre étant la signature des atomes ou des molécules, tout ou partie de celui-ci sera nécessaire pour "cartographier" une espèce atomique ou moléculaire répartie dans un certain milieu. Un exemple simple nous est fourni par l'astronomie : en regardant à l'aide d'un télescope une nébuleuse à travers un filtre interférentiel centré sur la raie $\mathrm{H}_{\alpha}$ de l'hydrogène atomique on voit directement les régions contenant de l'hydrogène atomique excité. Ce cas est particulièrement simple car l'identification de $\mathrm{H}$ ne nécessite qu'un élément spectral. Très souvent c'est tout un domaine spectral qu'il faudra examiner en chaque pixel et à plus haute résolution spectrale que celle donnée par le filtre interférentiel. Il faut alors concevoir un dispositif spécial : l'imageur spectral.

Outre l'astronomie, cette technique concerne l'observation de la terre depuis l'espace (le système imageur est du type télescope) [1] et la physico-chimie des surfaces (le système imageur est du type microscope) [2].

A. Girard a établi une classification prospective des imageurs spectraux incluant la spectroscopie par transformation de Fourier [3]. Nous resterons ici dans le cadre de limage 2D et de la spectroscopie dispersive. La réception de l'image et/ou du spectre est assurée par une matrice de $\mathrm{N}^{2}$ récepteurs. L'image ou le spectre obtenu sera donc intégré sur la largeur $\mathrm{r}$ des récepteurs et échantillonné avec un pas égal à r. Nous parlerons donc de pixels et d'éléments spectraux (e.s.).

Nous distinguerons trois cas suivant que l'on examine :

1- $\mathrm{N}^{2}$ pixels sur le même e.s. Cette situation est identique à celle où l'on employait un filtre interférentiel mais ici le filtrage est effectué à l'aide d'un monochromateur.

2- $\mathrm{N}$ pixels en ligne et $\mathrm{N}$ e.s. par pixels. Ce cas est parfois appelé spectroscopie 2D.

3- $\mathrm{N}^{2}$ pixels et $\mathrm{N}$ e.s. par pixel grâce à un multiplexage temporel. Il s'agit de la technique de mélanges codés de spectres.

Enfin nous comparerons ces méthodes du point de vue du rapport signal sur bruit.

\section{Monochromateur-imageur}

Il permet d'obtenir une image de $\mathrm{N}^{2}$ pixels dans une bande spectrale étroite. Nous nous limiterons ici au cas du microscope associé à un monochromateur à réseaux. Ce montage correspond au microscope Raman ou de fluorescence [2].

Deux montages sont possibles :

1- L'image est formée sur la fente d'entrée $F_{E}$ du monochromateur (fig.1). Le montage doit comporter un monochromateur double à dispersions soustractives car à la sortie du $1^{\text {er }}$ monochromateur l'image est brouillée, chaque tranche spectrale de largeur $\mathrm{d}_{\lambda}$ donnant une image décalée par rapport à celle qui correspond à la tranche précédente. Le 2 ème monochromateur recentre ces différentes images. La matrice de $\mathrm{N}^{2}$ détecteurs est placée sur la fente de sortie. Il lui correspond $\mathrm{N}^{2}$ pixels entrée sur $\mathrm{F}_{\mathrm{E}}$. Le grandissement de l'objectif du microscope est choisi en fonction du champ ou de la résolution de lobjet qui est souhaitée. 
La fente intermédiaire $F_{1}$ impose la résolution spectrale. Si la largeur d'un pixel dans le plan de $\mathrm{F}_{\mathrm{B}}$ est $\mathrm{r}$ et la largeur de $\mathrm{F}_{\mathrm{I}}: \mathrm{M}$ $r$, la fonction de transmission est une fonction trapèze dont la largeur à mi-hauteur est M r. Du premier au dernier pixel d'une ligne cette fonction est décalée de (N-1)r (fig.2). La partie commune du plateau sommital a une largeur égale à $\mathrm{p}=(\mathrm{M}-\mathrm{N}) \mathrm{r}$. $\mathrm{Si}$ l'on veut que cette largeur soit très supérieure au décalage il faut $\mathrm{M} \gg \mathrm{N}$.

Application numérique

$\mathrm{r}=10 \mu \mathrm{m} \mathrm{N}=250$.

Dispersion des réseaux : $1 \mathrm{~nm} / \mathrm{mm}$.

Largeur de $\mathrm{F}_{\mathrm{E}}: 2,5 \mathrm{~mm}$.

On veut : $\mathrm{p}=1 \mathrm{~nm}$. II lui correspond (M$N) r=1 \mathrm{~mm}=100 \mathrm{r}$.

D'où : $M=350$. La largeur de $F_{1}$ est $3,5 \mathrm{~mm}$.

La présence d'aberrations dans les montages classiques de spectromètre limite les performances de ce dispositif. Nous avons représenté sur la figure 1 un double monochromateur à réseau holographique concave corrigé des aberrations. Mais ces réseaux présentent l'inconvénient d'être peu dispersifs et donc d'admettre une étendue de faisceau faible à résolution donnée.

Il faut aussi que la largeur de la tâche

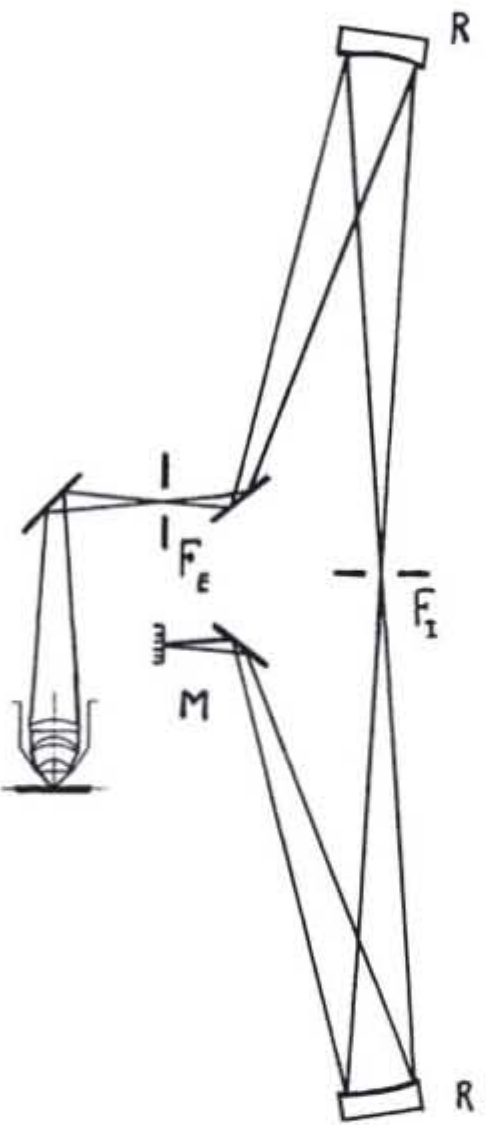

Fig. 1 de diffraction correspondant à la dimension du réseau soit inférieure à r.

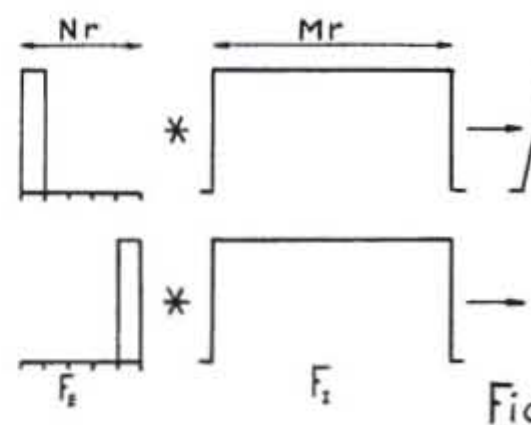

Fig 2 
2- L'image est formée sur le réseau (fig.3 extraite de la réf.2). L'échantillon est éclairé par un faisceau laser tournant. Son image $S_{1}$ est au foyer de la lentille $L_{1} . S_{2}$ est donc formée au foyer de $L_{2}$, sur le réseau. $L_{3}$ conjugue les deux réseaux. $L_{4}$ envoie $S_{3}$ sur le détecteur dimage $S_{4}$

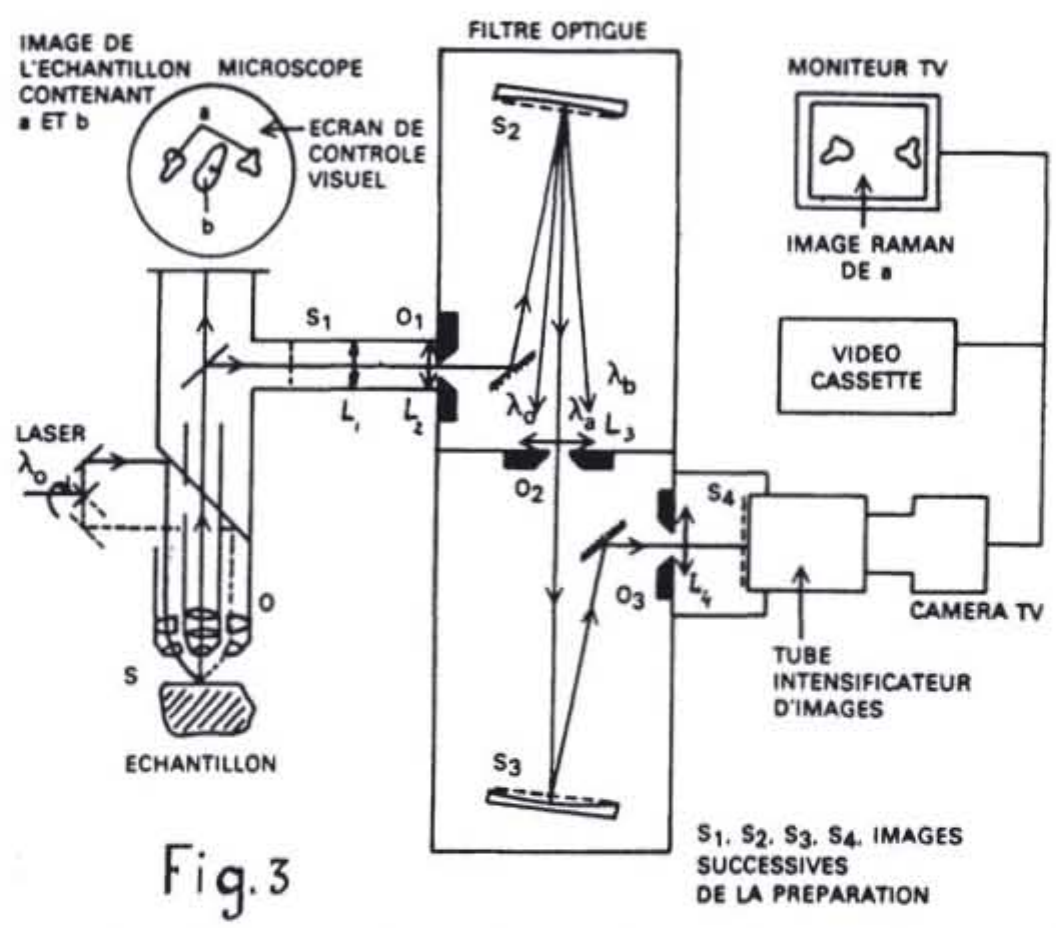

Ce système nécessite un grandissement important d'une part. D'autre part la tâche de diffraction produite par la fente d'entrée peut limiter la qualité de limage. Considérons le dispositif de la figure 4 qui permet un raisonnement plus simple. La source est projetée sur le réseau par la lentille L et le collimateur $\mathrm{C}$.

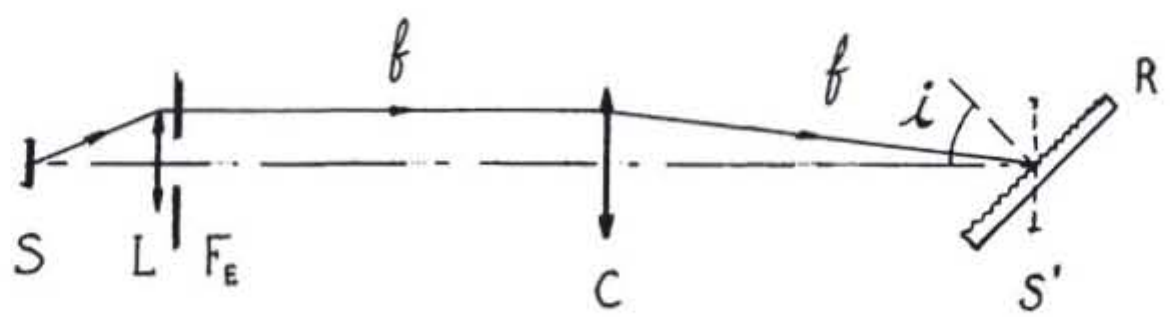

Fig. 4 
La largeur de la tâche de diffraction produite en $S^{\prime}$ est :

$\delta \mathrm{x}=\frac{\lambda}{\mathrm{e}} . \mathrm{f} . \quad \mathrm{e}: \mathrm{largeur}$ de la fente. $\mathrm{f}:$ distance focale de $\mathrm{C}$.

Or $: \frac{e}{f}=D \cdot \delta \lambda ; D:$ dispersion angulaire. $\delta \lambda:$ largeur spectrale de la fente.

D'où : $\quad \delta \mathrm{x} \cdot \delta \lambda=\frac{\lambda}{\mathrm{D}}$

Si le spectromètre est en montage Littrow (quasi autocollimation) : $D=\frac{2 \text { tgi }}{\lambda}$

i : angle d'incidence sur le réseau. Alors $\delta x . \delta \lambda=\frac{\lambda^{2}}{2 \text { tgi }}$

Application mumérique

i $6^{\circ}$ tgi=0,1 $\lambda=1 \mu \mathrm{m} ; \delta \lambda=10 \mathrm{~nm} \rightarrow \delta \mathrm{x}=0,5 \mathrm{~mm}$.

Si la largeur du réseau est $5 \mathrm{~cm}$, l'image ne contient que 100 points résolus dans sa largeur. Pour $\mathrm{i} \sim 45^{\circ}$ (tgi $=1$ ) on aurait 1000 points.

\section{Spectroscopie 2D}

Cette technique est employée pour l'observation de la terre depuis l'espace. Son schéma optique est donné par la figure 5. Un système télescopique forme l'image d'une bande étroite au sol sur la fente d'entrée $\mathrm{F}_{\mathrm{E}}$ d'un spectromètre. Dans ce plan la largeur des pixels est donc celle de $\mathrm{F}_{\mathrm{E}}$. Le spectre de chaque pixel est dispersé perpendiculairement à la fente et recueilli sur une matrice de récepteur placée dans le plan focal de l'objectif de sortie. La distance focale de ce dernier est choisie de manière à adapter la taille des pixels à celle des récepteurs. La résolution image et la résolution spectrale peuvent donc être choisies à volonté. On explore le sol ligne par ligne successivement dans le temps par basculement du miroir plan P.

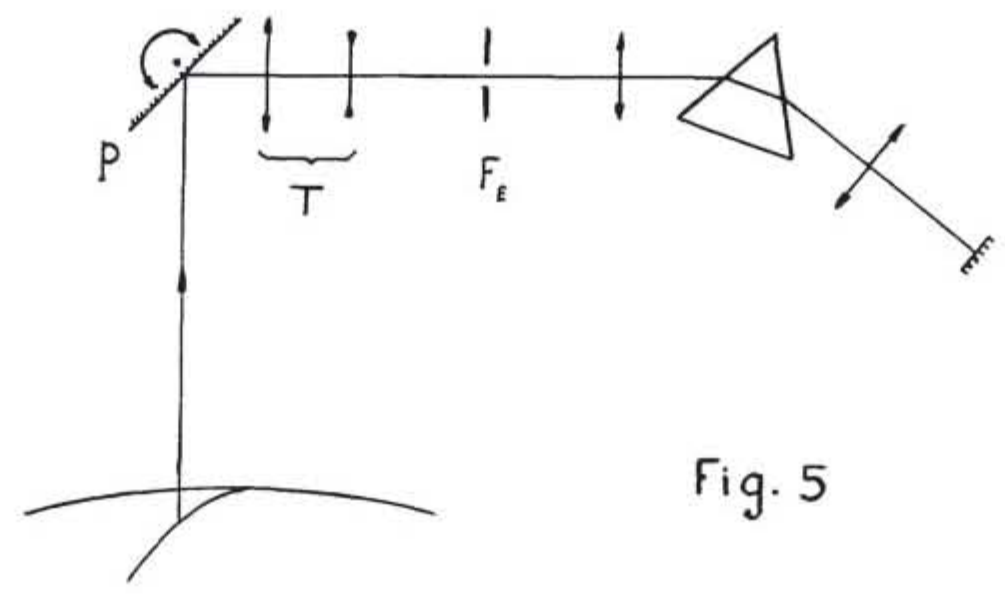


Sur la figure 5 nous avons représenté une optique réfractive. Mais en pratique on utilise une optique à miroirs (achromatisme) et un spectromètre à réseau. Les miroirs sont utilisés en dehors de l'axe ce qui génère des aberrations qu'il faut corriger par des combinaisons de miroirs. Un exemple est donné par l'appareil appelé ROSIS (Reflectivè Optics Imaging Spectrometer) [1]. Le télescope et le collimateur imageur du spectromètre sont du type "Blechinger". Ici encore le réseau est peu incliné $\left(2^{\circ}\right)$ et donc peu dispersif.

\section{Mélanges codés de spectres}

Le dispositif est le suivant : l'image est formée sur la fente d'entrée $\mathrm{F}_{\mathrm{E}}$ comme au cas 1. La fente de sortie $F_{S}$ du spectromètre est remplacée par une mosaïque de $\mathrm{N}^{2}$ récepteurs (fig.6).
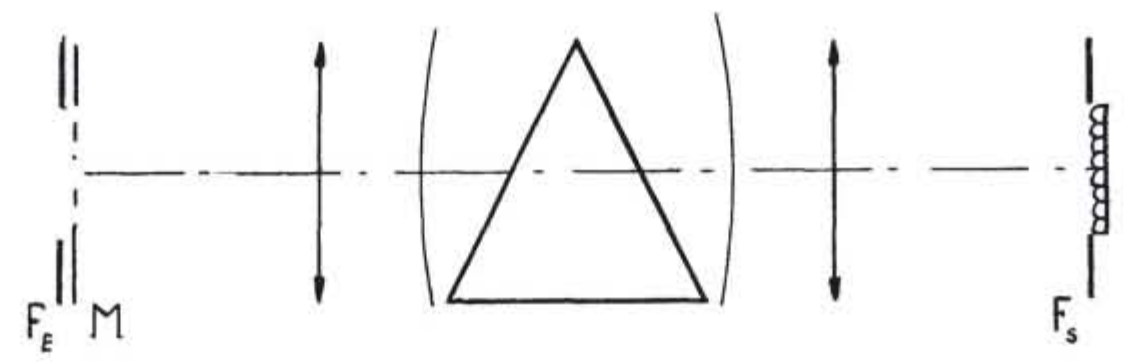

$$
\text { Fig. } 6
$$

A chaque pixel entrée correspond un spectre dans le plan de $\mathrm{F}_{\mathrm{S}}$. Les $\mathrm{N}$ pixels entrée d'une ligne image produisent donc $\mathrm{N}$ spectres qui se recouvrent suivant la direction de cette ligne. Nous raisonnerons donc sur une ligne, chaque ligne pouvant être traitée de la même façon.

Les différents éléments spectraux qui tombent sur un récepteur seront détectables s'ils sont modulés dans le temps selon un certain code. Ceci peut se faire grâce à des masques qui sont placés successivement sur $\mathrm{F}_{\mathrm{E}}$ (fig.5). Un traitement du signal à postériori permet de décoder le spectre et ainsi de le restituer. Nous allons pour cela nous inspirer de la spectroscopie par transformation de Hadamard [4].

Prenons l'exemple de $\mathrm{N}=3$ (fig.7). Sur la ligne de récepteurs chaque spectre est décalé de la largeur d'un récepteur par rapport au précédent, les éléments spectraux de même numéro correspondant à la même $\lambda$.

Appelons $\mathrm{L}_{\mathrm{ij}}$ l'élément spectral $\mathrm{n}^{\circ} \mathrm{j}$ émis par le pixel i et considérons le signal $\mathrm{S}_{\mathrm{k} 2}$ reçu par le récepteur $\mathrm{n}^{\circ} 2$ lorsque le masque $\mathrm{n}^{\circ} \mathrm{k}$ est placé à l'entrée.

$$
\begin{array}{ll}
-\mathrm{k}=1 \text { : pixel } \mathrm{n}^{\circ} 1 \text { masqué : } & 0+\mathrm{L}_{23}+\mathrm{L}_{32}=\mathrm{S}_{12} \\
-\mathrm{k}=2 \text { : pixel } \mathrm{n}^{\circ} 2 \text { masqué : } & \mathrm{L}_{14}+0+\mathrm{L}_{32}=\mathrm{S}_{22} \\
-\mathrm{k}=3 \text { : pixel } \mathrm{n}^{\circ} 3 \text { masqué : } & \mathrm{L}_{14}+\mathrm{L}_{23}+0=\mathrm{S}_{32}
\end{array}
$$


La résolution du système donne :

$$
\begin{aligned}
& \mathrm{L}_{14}=1 / 2\left(-\mathrm{S}_{12}+\mathrm{S}_{22}+\mathrm{S}_{32}\right) \\
& \mathrm{L}_{23}=1 / 2\left(\mathrm{~S}_{12}-\mathrm{S}_{22}+\mathrm{S}_{32}\right) \\
& \mathrm{L}_{32}=1 / 2\left(\mathrm{~S}_{12}+\mathrm{S}_{22}-\mathrm{S}_{32}\right)
\end{aligned}
$$

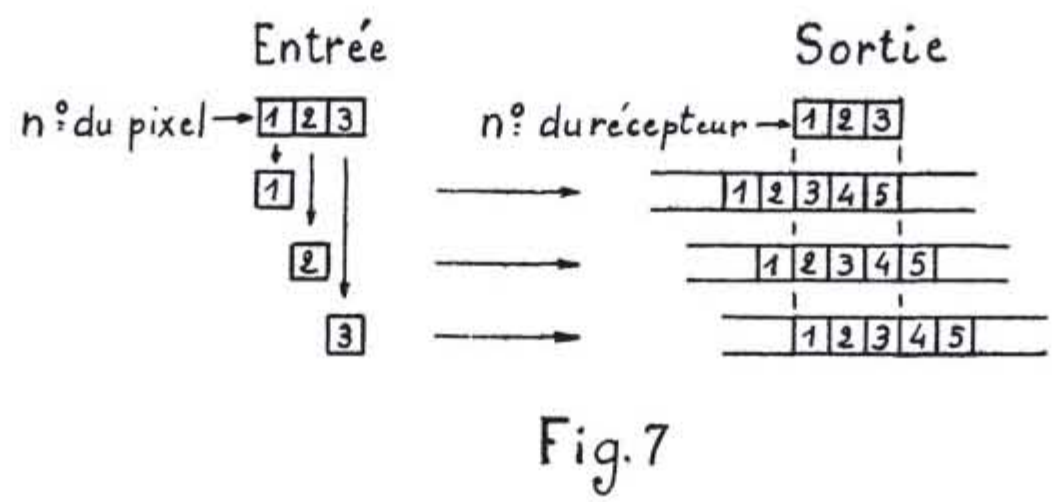

Les pixels $1,2,3$, et leurs spectres sont représentés décalés dans la direction perpendiculaire à la dispersion pour plus de clarté. Chaque récepteur reçoit les e.s. placés dans sa colonne. Par exemple le récepteur $\mathrm{n}^{\circ} 1$ reçoit les e.s. 3,2 et 1 .

Il en est de même pour les deux autres récepteurs. Plus généralement la fonction de transmission du masque $\mathrm{j}$ est une fonction binaire $\mathrm{t}_{\mathrm{j} p}$ composée de $\mathrm{N}$ éléments $(\mathrm{p}=1 \mathrm{a} \mathrm{N}$ ) de même largeur qui sont soit nuls (éléments opaques) soit égaux à 1 (éléments transparents). Le code est changé $\mathrm{N}$ fois.

Dans l'exemple précédent $(\mathrm{N}=3)$ les codes étaient successivement : $011 ; 101 ; 110$.

Pour $\mathrm{N}=7$ les codes seront par exemple : 0101110 et ses permutations circulaires.

Dans le plan de sortie, pour le masque j l'éclairement est de la forme :

$E_{j}(x)=\sum_{p=1}^{N} t_{j p} L_{p}(x-(p-1) r)$

$\mathrm{x}$ : abscisse dans la direction de la dispersion du spectre; $\mathrm{r}$ : largeur du récepteur.

Pour l'ensemble des $\mathrm{N}$ opérations :

$\left(\begin{array}{l}E_{1}(x) \\ E_{j}(x) \\ E_{N}(x)\end{array}\right)=\left(\begin{array}{l}t_{j p} \\ \end{array}\right)\left(\begin{array}{l}L_{1}(x) \\ L_{j}(x-(j-1) r) \\ L_{N}(x-(N-1) r)\end{array}\right)$

et par inversion on obtient les différents spectres $\mathrm{L}_{\mathrm{j}}(\mathrm{x})$. En réalité le signal délivré par la matrice est proportionnel à l'éclairement $\mathrm{E}_{\mathrm{j}} \mathrm{x}$ ) convolué par une fonction rectangle de largeur $\mathrm{r}$ et échantillonné avec un pas égal à $\mathbf{r}$. Les spectres restitués sont donc aussi convolués avec la fonction d'appareil et échantillonnés avec un pas égal à r. 
La matrice de codage $\left(\mathrm{t}_{\mathrm{jp}}\right)$ doit être inversible et procurer le gain multiplex en rapport signal sur bruit. Une matrice binaire $S$, dérivée des matrices de Hadamard est bien adaptée à ce problème [4]. Son inverse est une matrice dont les éléments sont des +1 ou des -1 et :

$$
L_{j}(x-(j-1) r)=\frac{2}{N+1} \sum_{p=1}^{N} t_{j p}^{-1} E_{p}(x)
$$

Le coefficient de remplissage des matrices $S$ (nombre de 1/nombre d'éléments) est voisin de 0,5 . Les signaux "primaires" sont donc en moyenne $N / 2$ fois plus important que lorsqu'il n'y a qu'une seule fente. Si le bruit dominant est un bruit indépendant du signal le rapport signal sur bruit est aussi plus important. Mais il faut encore que ce gain sur le rapport signal sur bruit des signaux primaires produise un gain sur le rapport signal sur bruit des éléments spectraux restitués. Nous verrons plus loin que c'est le cas avec les matrices $S$.

\section{Rapports signal sur bruit}

Nous allons comparer ces trois techniques du point de vue du rapport signal sur bruit. Pour cela nous supposons que dans les trois cas on dispose du même temps total $\mathrm{T}$ pour explorer les éléments spectraux des $\mathrm{N}^{2}$ pixels.

\section{1-Monochromateur-imageur :}

On obtient $\mathrm{N}^{2}$ pixels dans un élément spectral. Il faut changer d'e.s. $\mathrm{N}$ fois. Pour chaque opération le temps d'intégration du flux sur 1 e.s. est : $\mathrm{T} / \mathrm{N}$.

2-Spectroscopie $2 D$ :

On explore limage ligne par ligne. Pour chaque opération le temps d'intégration du flux sur 1 e.s. est T/N. Les techniques 1 et 2 ont donc même expression pour le rapport signal sur bruit sur 1 e.s.

\section{3- Mélanges codés de spectres:}

Cette technique nécessite aussi $N$ opérations. Le temps d'intégration du flux pour un signal $E_{j}$ est donc $\mathrm{T} / \mathrm{N}$.

Nous supposons que le bruit $\Delta \mathrm{E}$ est le même pour tous les $\mathrm{E}_{\mathrm{j}}$. Alors d'après la formule d'inversion le bruit sur l'e.s. $\mathrm{L}_{\mathrm{i}}$ est :

$$
\Delta \mathrm{L}=\frac{2}{\mathrm{~N}+1} \cdot \Delta \mathrm{E} \cdot\left[\sum_{\mathrm{p}=1}^{\mathrm{N}}\left(\mathrm{t}_{j \mathrm{p}}^{-1}\right)^{2}\right]^{1 / 2}=\frac{2}{\mathrm{~N}+1} \cdot \Delta \mathrm{E} \cdot \mathrm{N}^{\mathrm{s} /}
$$

D'où le rapport signal sur bruit: $\frac{s}{b}=\frac{L_{i}}{\Delta E} \cdot \frac{N^{1 / 2}}{2}$

Nous distinguerons deux cas :

a) Régime de bruit propre de détecteur (I.R.) :

Pour les techniques 1 et 2 on $a: \frac{s}{b}=\frac{L_{i}}{\Delta E}$ D'où le gain : $G_{3 / 1,2}=\frac{N^{1 / 2}}{2}$

b) Régime de bruit quantique (V, U.V.) 
Soit $\mathbf{L}$ la valeur moyenne des $L_{j} . \sum_{i} L_{i}=N L$ Alors : $E_{j} \# \frac{N}{2} L$ et $\Delta E_{j}=\left(\frac{N}{2} L\right)^{1 / 2}$

D'où :

$\frac{\mathrm{s}}{\mathrm{b}}=\frac{\mathrm{L}_{\mathrm{i}}}{\left(\frac{\mathrm{N}}{2} \mathrm{~L}\right)^{1 / 2}} \cdot \frac{\mathrm{N}^{1 / 2}}{2}=\frac{1}{\sqrt{2}} \cdot \frac{\mathrm{L}_{\mathrm{i}}}{(\mathrm{L})^{1 / 2}}$

Pour les techniques 1 et 2 on a: $\frac{s}{b}=\frac{L_{i}}{L_{i}^{1 / 2}}$

D'où le gain : $G_{3 / 1,2}=\frac{1}{\sqrt{2}}\left(\frac{L_{i}}{L}\right)^{1 / 2}$

$\mathrm{G}$ dépend de l'e.s. $\mathrm{L}_{\mathrm{i}}$ considéré. Le gain est appréciable dans le cas d'un spectre de raies peu nombreuses $\left(L_{i}>>L\right)$. Mais il tombe à la valeur 1 partout pour un spectre continu quasi constant $\left(\mathrm{L}_{\mathrm{i}} \# \mathbf{L}\right)$,

Cette technique n'a pas encore, à ma connaissance, donné lieu à des applications concrètes dans le domaine de l'imagerie spectrale.

Un cas particulier intéressant est celui où les pixels d'entrée émettent le même spectre c'est-àdire lorsqu'une source homogène est projetée sur l'entrée du spectromètre. Celui-ci devient un spectromètre à grille "dynamique". Si p est le nombre d'élément du code $(\mathrm{p}<\mathrm{N})$ le gain multiplex correspondant est $\mathrm{G}=(1 / 2) \mathrm{p}^{1 / 2}[5]$.

\section{Conclusion}

Nous avons traité le problème général consistant à obtenir $\mathrm{N}$ éléments spectraux par pixel pour $\mathrm{N}^{2}$ pixels, $\mathrm{N}^{2}$ étant le nombre des récepteurs d'une matrice. Pour cela nous avons comparé du point de vue rapport signal sur bruit trois techniques d'imagerie spectrale dispersive. Les deux premières sont équivalentes tandis que la troisième procure un gain par rapport aux deux autres. Cependant pour cette dernière le domaine spectral obtenu n'est pas le même pour tous les pixels d'une ligne (voir fig.7)

En pratique de difficiles problèmes d'aberrations sont à résoudre. Pour les montages 1 et 2 si l'on emploie un spectromètre à réseau plan en montage Czerny-Turner il apparaît un astigmatisme important. Alors un codage par masques du réseau permet une résolution sub-aberration [5][6][7].

\section{Références}

[1] Proceeding on Imaging spectroscopy of the terrestrial environment ; SPIE 1298 (April 1990, Orlando, Florida)

[2] Microsonde mole; Fiche technique Jobin-Yvon.

La résolution temporelle et la résolution spatiale en spectroscopie Raman ; M. Delhaye, M. Bridoux, F. Wallart ; Le Courrier du CNRS janv. 81.

Bioimaging and two-dimensional spectroscopy ; SPIE 12054 (January 1990, Los Angeles). 
[3] Classification des imageurs spectraux ; A. Girard ; J. Optics (Paris) 15, 7 (1984.

[4] Modulations techniques in spectrometry; M. Harwit, J.A. Decker ; chap.III du livre : Progress in optics XII, North Holland 1974.

[5] Le codage de la fonction d'appareil dans les spectroscopies de multiplexage ; R. Damaschini ; OPTO 90 , p.73, E.S.I. Publications.

[6] Sub-aberration image résolution ; $\mathrm{ECO}_{2}$ Paris avril 89 ; SPIE 1135 , Image Processing III.

[7] Résolution sub-aberration d'une image par codage de la pupille du système optique; A. Salloun et R. Damaschini ; OPTO 90, p.511, ESI Publications. 